\title{
Classifying Non-periodic Sequences by Permutation Transducers
}

\author{
Hans Zantema ${ }^{1,2(\bowtie)}$ and Wieb Bosma ${ }^{2,3}$ \\ 1 Department of Computer Science, TU Eindhoven, \\ P.O. Box 513, 5600 MB Eindhoven, The Netherlands \\ h.zantema@tue.nl \\ 2 Radboud University Nijmegen, \\ P.O. Box 9010, 6500 GL Nijmegen, The Netherlands \\ w. bosma@math.ru.nl \\ 3 Centrum voor Wiskunde en Informatica, Amsterdam, The Netherlands
}

\begin{abstract}
Transducers order infinite sequences into natural classes, but permutation transducers provide a finer classification, respecting certain changes to finite segments. We investigate this hierarchy for non-periodic sequences over $\{0,1\}$ in which the groups of 0 s and 1s grow according to simple functions like polynomials. In this hierarchy we find infinite strictly ascending chains of sequences, all being equivalent with respect to ordinary transducers.
\end{abstract}

\section{Introduction}

Equivalence under transducers organizes infinite sequences into a hierarchy with interesting properties, as ongoing research is revealing, see for example $[3,6]$ and the conference paper [5] at DLT 2016. In this setting the main definition is that for two sequences $\sigma, \tau$ we have that $\sigma \geq \tau$ if and only if there exists a transducer $T$ that produces $\tau$ when consuming $\sigma$. Here a transducer is a deterministic automaton producing output strings on every transition. Two sequences $\sigma, \tau$ are called equivalent, notation $\sigma \sim \tau$ if both $\sigma \geq \tau$ and $\tau \geq \sigma$. A straightforward construction shows that $\sigma \sim u \sigma$ for any sequence $\sigma$ and any finite string $u$, so prepending or removing a finite initial word remains inside the class. The pre-order $\geq$ gives rise to an order on the equivalences classes of $\sim$; the bottom element in this order consists of the class of ultimately periodic sequences.

In the current paper we investigate a more fine-grained hierarchy on sequences based on an alternative pre-order $\geq_{p}$. Here prepending or removing initial segments may change the class, but other basic properties are kept, like $\sigma \geq_{p} h(\sigma)$ for any morphism $h$. The idea is that we add the requirement that transducers should be permutation transducers. This means that not only for every state and symbol there is exactly one outgoing arrow (as is required by determinism), but also exactly one incoming arrow: it will thus be a permutation automaton (see $[1,7,8]$ ) with output, just like a finite state transducer is a DFA with output. Our original motivation for permutation transducers was to be able 
to compare and classify two-sided sequences as was elaborated in [2]. There we already made some first investigations on ordering (one-sided) sequences by permutation transducers, raising several issues that we worked out in the current paper.

So we define $\sigma \geq_{p} \tau$ if and only if a permutation transducer $P$ exists such that $P(\sigma)=\tau$, and $\sigma \sim_{p} \tau$ if and only if both $\sigma \geq_{p} \tau$ and $\tau \geq_{p} \sigma$. In [2] we already showed that $0^{\omega} \varkappa_{p} 10^{\omega}$, a clear illustration that initial segments matter in this context. Again the pre-order $\geq_{p}$ on sequences gives rise to an order on $\sim_{p^{-}}$ equivalence classes; here the bottom element is the class of all periodic sequences. In [2] we showed that the ultimately periodic sequences that are not periodic form an atomic class. Here the focus is on sequences that are not ultimately periodic. In particular, we look at sequences of the shape

$$
\langle f\rangle=10^{f(0)} 10^{f(1)} 10^{f(2)} \cdots \text { and } \llbracket f \rrbracket=0^{f(0)} 1^{f(1)} 0^{f(2)} \cdots,
$$

for various functions $f: \mathbb{N} \rightarrow \mathbb{N}$, in particular polynomials. Based on ordinary transducers one has $\langle f\rangle \sim \llbracket f \rrbracket$ if $f(n)>0$ for all $n \in \mathbb{N}$, and for all linear $f, g$ it holds $\langle f\rangle \sim\langle g\rangle$. A main result of [4] states that the class containing the sequences of the shape $\langle f\rangle$ for $f$ linear is atomic, that is, if $\langle f\rangle \geq \sigma$ for $f$ linear, then either $\sigma \geq\langle f\rangle$ or $\sigma$ is ultimately periodic. In [3] it was shown that a similar result holds for quadratic functions, while in $[5,6]$ it was shown that for higher degree it does not hold. Here we are interested in considering $\geq_{p}$ and $\sim_{p}$ instead.

In [2] we already showed that $\langle f\rangle \sim_{p}\langle g\rangle$ for $f, g$ linear. Here we show that the corresponding class is not atomic: we show that for ascending $f$ we have $\langle f\rangle \geq_{p} \llbracket f \rrbracket$ but not the other way around, and we even show that the class containing $\langle f\rangle$ for linear $f$ is an upper bound of infinitely many distinct classes, in particular

$$
\llbracket n \rrbracket<_{p} \llbracket n+2 \rrbracket<_{p} \llbracket n+4 \rrbracket<_{p} \llbracket n+8 \rrbracket<_{p} \llbracket n+16 \rrbracket<_{p} \cdots \leq_{p}\langle n\rangle .
$$

We write $\sigma<_{p} \tau$ if $\tau \geq_{p} \sigma$ but not $\sigma \geq_{p} \tau$, and use $\llbracket f(n) \rrbracket$ as shorthand for $\llbracket n \mapsto$ $f(n) \rrbracket$, and similarly for $\langle\cdot\rangle$. While all $\langle f\rangle$ for $f$ quadratic are equivalent under ordinary transduction, we show that this does not hold for $\sim_{p}$; in particular, we obtain the infinite ascending chain

$$
\left\langle(n+1)^{2}\right\rangle<_{p}\left\langle n^{2}\right\rangle<_{p}\left\langle(n-1)^{2}\right\rangle<_{p}\left\langle(n-2)^{2}\right\rangle<_{p}\left\langle(n-4)^{2}\right\rangle<_{p}\left\langle(n-8)^{2}\right\rangle<_{p} \cdots .
$$

Typically, for proving $\sigma<_{p} \tau$ the easier part is giving an explicit permutation transducer $P$ satisfying $P(\tau)=\sigma$. The hard part is showing that a permutation transducer for the other way around does not exist. For instance, the easy part of showing $\llbracket n \rrbracket<_{p} \llbracket n+2 \rrbracket$ can be done using the following permutation transducer, proving $\llbracket n+2 \rrbracket \geq_{p} \llbracket n \rrbracket$. In presenting a transducer by a picture an arrow labeled by $a \mid u$ means that an input symbol $a$ is consumed and the string $u$ is

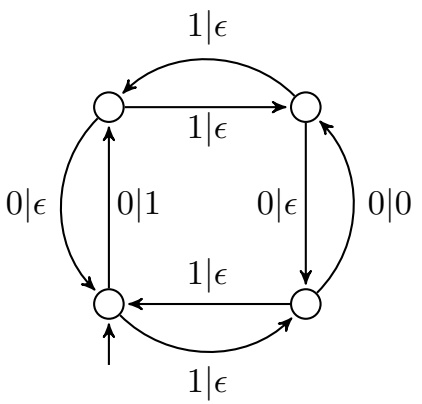


produced as output. The initial state is indicated by an incoming arrow not starting in a state. When consuming $\llbracket n+2 \rrbracket=0^{2} 1^{3} 0^{4} 1^{5} \ldots$ by this permutation transducer indeed $\llbracket n \rrbracket=10^{2} 1^{3} 0^{4} \ldots$ is produced. Here the two 1 -arrows between the two top states are never used, and may also produce anything else.

An even simpler ordinary transducer doing the same job, but which is not a permutation transducer, is easily found, as $\llbracket n \rrbracket$ can also be obtained from $\llbracket n+2 \rrbracket$ by simply putting a single symbol 1 in front.

The remaining proof obligation, that no permutation transducer exists transforming $\llbracket n \rrbracket$ to $\llbracket n+2 \rrbracket$, is much harder. For doing this we investigate the pattern of any sequence that can be obtained by applying a permutation transducer to $\llbracket n \rrbracket$, and then prove that $\llbracket n+2 \rrbracket$ does not satisfy this pattern. For all other claims in this paper containing ' $<_{p}$ ' we give similar arguments all being instances of the following three cases. The first case investigates the creation of isolated $1 \mathrm{~s}$, leading to $\llbracket f \rrbracket<_{p}\langle f\rangle$ for ascending $f$. The second one investigates transducts of $\langle f\rangle$ and $\llbracket f \rrbracket$ for those $f$ (such as $f(n)=n !)$ for which for every $m$ there exists $N$ such that $f(n) \equiv 0 \bmod m$ whenever $n>N$. The third one investigates transducts of $\langle f\rangle$ and $\llbracket f \rrbracket$ for $f$, such as polynomials, for which $n \mapsto f(n) \bmod m$ is periodic for every $m$.

We consider four basic ways to transform functions $f: \mathbb{N} \rightarrow \mathbb{N}$ : transforming $f(n)$ to $f(n)+k$ and to $f(n+k)$ for any $k \geq 1$, and to $k f(n)$ and to $f(k n)$ for any $k>1$. For all of them we investigate how $\langle f(n)\rangle$ and $\llbracket f(n) \rrbracket$ relate to their transformed variants, both with respect to ordinary transducers and permutation transducers.

The paper is organized as follows. We start by preliminaries in Sect.2. In Sect. 3 we classify permutation transducts of particular sequences $\sigma$, in order to be able to prove $\sigma \nsupseteq_{p} \tau$ for certain $\tau$. In Sect. 4 we investigate the effect of transforming $f$ in the above given four ways. In Sect. 5 we investigate $\llbracket f \rrbracket$ and $\langle f\rangle$ for linear functions $f$; in particular, we give an infinite strictly ascending chain of them. In Sect. 6 we investigate polynomials of higher degree; in particular, we give an infinite strictly ascending chain of sequences $\langle f\rangle$ for quadratic polynomials $f$. Due to lack of space some proofs are omitted in this paper; they can be found in the full version [9].

\section{Preliminaries}

In the following we assume $\Sigma=\{0,1\}$.

Definition 1. A finite state transducer $T=\left(Q, q_{0}, \delta, \lambda\right)$ consists of a finite set $Q, q_{0} \in Q, \delta: Q \times \Sigma \rightarrow Q, \lambda: Q \times \Sigma \rightarrow \Sigma^{*}$. For $\sigma: \mathbb{N} \rightarrow \Sigma$ we define $T(\sigma)=\lambda\left(q_{0}, \sigma(0)\right) \lambda\left(q_{1}, \sigma(1)\right) \lambda\left(q_{2}, \sigma(2)\right) \cdots$ for $q_{i}$ defined by $q_{i+1}=\delta\left(q_{i}, \sigma(i)\right)$ for $i \geq 0$.

A permutation transducer over $\Sigma$ is a finite state transducer $T=\left(Q, q_{0}, \delta, \lambda\right)$ with the additional requirement that for every $a \in \Sigma$ the function $q \mapsto \delta(q, a)$ is a bijection from $Q$ to $Q$.

For $\sigma, \tau: \mathbb{N} \rightarrow \Sigma$ we define $\geq_{p}, \sim_{p}$ and $>_{p}$ by

$$
\sigma \geq_{p} \tau \Longleftrightarrow \exists \text { permutation transducer } T: \tau=T(\sigma) \text {, }
$$




$$
\sigma \sim_{p} \tau \Longleftrightarrow \sigma \geq_{p} \tau \wedge \tau \geq_{p} \sigma, \quad \sigma>_{p} \tau \Longleftrightarrow \sigma \geq_{p} \tau \wedge \neg\left(\tau \geq_{p} \sigma\right) .
$$

In drawing pictures for transducers we write an arrow from $p$ to $q$ labeled by $a \mid u$ if $\delta(p, a)=q$ and $\lambda(p, a)=u$. We use $\geq, \sim,>$ for the similar relations on sequences based on ordinary finite state transducers, that is, without the additional bijectivity requirement. These were studied extensively in [3-6]. To see the effect of the additional requirement of permutation transducers, throughout the paper in presenting properties of $\geq_{p}, \sim_{p},>_{p}$ we often present the corresponding properties of $\geq, \sim,>$.

For a homomorphism $h: \Sigma \rightarrow \Sigma^{+}$the transducer $T_{h}=\left(\left\{q_{0}\right\}, q_{0}, \delta, \lambda\right)$ defined by $\delta\left(q_{0}, a\right)=q_{0}$ and $\lambda\left(q_{0}, a\right)=h(a)$ for all $a \in \Sigma$ is a permutation transducer satisfying $T_{h}(\sigma)=h(\sigma)$ for all $\sigma$, proving that $\sigma \geq_{p} h(\sigma)$. In particular, for choosing $h$ to be the identity we obtain that $\geq_{p}$ is reflexive. A straightforward construction given in [2] shows that $\geq_{p}$ is transitive. Hence $\geq_{p}$ is a pre-order, yielding a partial order on equivalence classes with respect to the equivalence relation $\sim_{p}$. By defining $h(a)=0$ for all $a \in \Sigma$ we obtain $T_{h}(\sigma)=0^{\omega}$ for every $\sigma$. Hence the equivalence class of $0^{\omega}$ is the bottom element in this order; it consists of all (purely) periodic sequences as was shown in [2].

A partial permutation transducer $T=\left(Q, q_{0}, \delta, \lambda\right)$ consists of a finite set $Q$ and initial state $q_{0} \in Q$, together with a partial function $\delta: Q \times \Sigma \rightarrow Q$ such that for every $q \in Q, a \in \Sigma$ there is at most one $q^{\prime} \in Q$ such that $\delta\left(q^{\prime}, a\right)=q$, and $\lambda: Q \times \Sigma \rightarrow \Sigma^{*}$ is a partial function that is defined on the same pairs that $\delta$ is defined for. Thus, in a permutation transducer for every symbol $a \in \Sigma$ there is exactly one incoming and exactly one outgoing $a$-arrow for every state $q \in Q$, but in a partial permutation transducer 'exactly one' is weakened to 'at most one'. As observed in [2], just like every partial permutation of a set can be extended to a permutation, every partial permutation transducer can be extended to a permutation transducer. Sometimes we will present a permutation transducer by only giving a partial permutation transducer and leaving the extension implicit.

From the introduction recall the definitions

$$
\langle f\rangle=\sigma_{f}=10^{f(0)} 10^{f(1)} 10^{f(2)} \ldots \text { and } \llbracket f \rrbracket=0^{f(0)} 1^{f(1)} 0^{f(2)} \ldots
$$

for any $f: \mathbb{N} \rightarrow \mathbb{N}$. For the latter it is natural to require $f(n)>0$ for all $n>0$, to avoid collapsing groups; we will say that $f$ is positive if it satisfies this property. Note that every sequence $\sigma$ that is not eventually constant has a natural representation $\llbracket f \rrbracket$ for some (positive) function $f$. The same is true for $\langle f\rangle$ if $\sigma(0)=1$, with $f$ usually not positive.

Writing $\langle f(n)\rangle$ for $\langle f\rangle$, we obtain $\langle n\rangle=11010010001 \cdots$, and $\langle n+1\rangle=$ $101001000 \cdots$, so $\langle n+1\rangle=\operatorname{tail}(\langle n\rangle)$. Using similar shorthand notation, $\llbracket n \rrbracket=$ $1^{1} 0^{2} 1^{3} 0^{4} 1^{5} \cdots$, and $\llbracket n+1 \rrbracket=0^{1} 1^{2} 0^{3} 1^{4} 0^{5} \cdots$, so $T_{h}(\llbracket n \rrbracket)=\llbracket n+1 \rrbracket$ and $T_{h}(\llbracket n+$ $1 \rrbracket)=\llbracket n \rrbracket$ for $h(0)=1, h(1)=0$, proving $\llbracket n \rrbracket \sim_{p} \llbracket n+1 \rrbracket$.

We continue with a fruitful lemma.

Lemma 2. Let $P$ be a permutation transducer over a finite alphabet $\Sigma$. Then there exists an integer $N>0$ such that for every state $q$ and every $u \in \Sigma^{+}$it holds that $\delta\left(q, u^{N}\right)=q$. 
Proof. Let $n$ be the number of states. Then $q \mapsto \delta(q, u)$ is a permutation on the $n$ states. Choose $N$ to be the least common multiple of all $k$ with $k \leq n$. Then $q \mapsto \delta\left(q, u^{N}\right)=(q \mapsto \delta(q, u))^{N}$ is the identity.

Proposition 3. For every positive function $f: \mathbb{N} \rightarrow \mathbb{N}$ holds

- $\langle f\rangle \sim \llbracket f \rrbracket$, and

- $\langle f\rangle \geq_{p} \llbracket f \rrbracket$.

Proof. This is proved by the following two transducers.
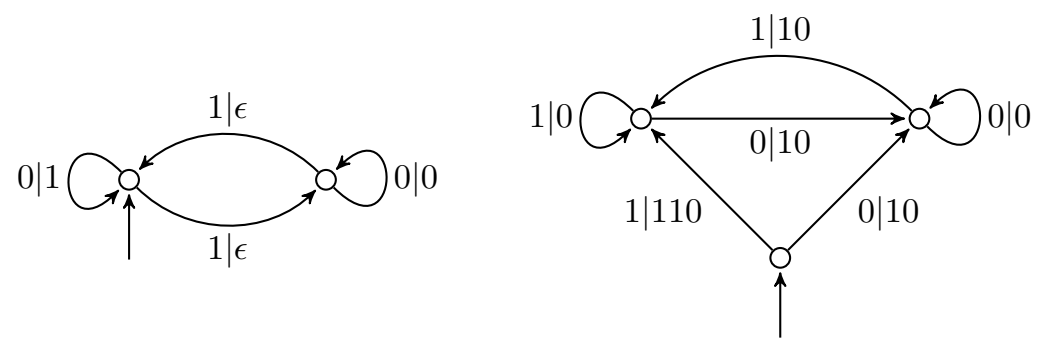

The left one is a permutation transducer replacing sequences of consecutive 0's that are demarcated by a single 1 , alternatingly by the same number of 0's or 1's; hence transforming $\langle f\rangle$ to $\llbracket f \rrbracket$, showing $\langle f\rangle \geq_{p} \llbracket f \rrbracket$ and thus also $\langle f\rangle \geq \llbracket f \rrbracket$.

The right one is an ordinary transducer (but not a permutation transducer) transforming $\llbracket f \rrbracket$ to $\langle f\rangle$, showing that $\llbracket f \rrbracket \geq\langle f\rangle$. Together with the just observed $\langle f\rangle \geq \llbracket f \rrbracket$ this proves $\langle f\rangle \sim \llbracket f \rrbracket$.

Now we show that $\llbracket f \rrbracket \geq_{p}\langle f\rangle$ does not generally hold: for certain $f$ no permutation transducer $P$ exists transforming $\llbracket f \rrbracket$ to $\langle f\rangle$. The key idea is that the isolated 1s in $\langle f\rangle$ can not be created when the input only contains big groups of 0 s and $1 \mathrm{~s}$ as in $\llbracket f \rrbracket$. In fact we prove the following stronger result.

Theorem 4. Let $f, g: \mathbb{N} \rightarrow \mathbb{N}$ satisfy $\lim _{n \rightarrow \infty} f(n)=\lim _{n \rightarrow \infty} g(n)=\infty$. Then no permutation transducer $P$ such that $P(\llbracket f \rrbracket)=\langle g\rangle$.

Proof. Assume such a $P=\left(Q, q_{0}, \delta, \lambda\right)$ exists. Use Lemma 2 to choose $p$ such that $\delta\left(q, 0^{p}\right)=\delta\left(q, 1^{p}\right)=q$ for all states $q$. Write $u(q, 0)=\lambda\left(q, 0^{p}\right)$ and $u(q, 1)=$ $\lambda\left(q, 1^{p}\right)$ for all states $q$. Since $\lim _{n \rightarrow \infty} f(n)=\infty$ a number $N$ exists such that $f(n)>2 p$ for all $n \geq N$. Hence beyond a finite initial part, the sequence $\llbracket f \rrbracket$ is composed of strings $0^{k}$ and $1^{k}$ for $k>2 p$. For each such string the permutation transducer $P$ produces a prefix of $u(q, i)^{\omega}$ that starts by $u(q, i)^{2}$ for some state $q$ and $i \in\{0,1\}$. Beyond a finite initial part, the resulting output $\langle g\rangle$ is the concatenation of such prefixes. Assume that one of the occurring strings $u(q, i)^{2}$ contains a symbol 1 . Then it contains at least two symbols 1 at distance at most $m$, where $m$ is the maximal size of all $u(q, i)$. Since $\lim _{n \rightarrow \infty} g(n)=\infty$, the total number of occurrences of $u(q, i)^{2}$ in $\langle g\rangle$ that contain a symbol 1 , is finite. This contradicts the fact that $\langle g\rangle$ contains infinitely many 1 s.

For proving more claims of the type $\sigma \nsupseteq_{p} \tau$ we typically investigate the shape of sequences $P(\sigma)$, the transducts of $\sigma$ : then it remains to show that $\tau$ is not of the required shape. In the next section we give a number of results of this type. 


\section{Classifying Transducts}

We want to classify permutation transducts of sequences of the shape $\langle f\rangle$ and $\llbracket f \rrbracket$ for well-known functions $f$, like polynomials. A key property of polynomials $f$ that will be exploited is that the function $n \mapsto(f(n) \bmod m)$ is periodic for every $m>0$. We start by a class of functions for which the analysis is slightly simpler, namely functions like $f(n)=n$ ! for which $n \mapsto(f(n) \bmod m)$ is ultimately 0 for every $m>0$.

Theorem 5. Let $f: \mathbb{N} \rightarrow \mathbb{N}$ be a positive function for which for every $m>0$ there exists $N \in \mathbb{N}$ such that $f(n) \equiv 0 \bmod m$ for all $n>N$. If $\llbracket f \rrbracket \geq p$ then there exist $u, c, d \in \Sigma^{*}$ and $b, h \in \mathbb{N}$ such that

$$
\sigma=u \prod_{i=0}^{\infty}\left(c^{f(b+2 i) / h} d^{f(b+2 i+1) / h}\right)=u c^{f(b) / h} d^{f(b+1) / h} c^{f(b+2) / h} d^{f(b+3) / h} \ldots
$$

Proof. Let $P=\left(Q, q_{0}, \delta, \lambda\right)$ be a permutation transducer such that $P(\llbracket f \rrbracket)=\sigma$. By Lemma 2 there exists $h$ such that $\delta\left(q, 0^{h}\right)=\delta\left(q, 1^{h}\right)=q$ for all $q$. Choose $b$ even such that $f(i) \equiv 0 \bmod h$ for all $i \geq b$. Let $u$ be the output of $P$ of the initial part $v=0^{f(0)} 1^{f(1)} \cdots 1^{f(b-1)}$, and let $q=\delta\left(q_{0}, v\right)$. Let $c=\lambda\left(q, 0^{h}\right)$ and $d=\lambda\left(q, 1^{h}\right)$. Then the next blocks $0^{f(b)}, 1^{f(b+1)}, 0^{f(b+2)}, 1^{f(b+3)}, \ldots$ produce output $c^{f(b) / h} d^{f(b+1) / h} c^{f(b+2) / h} d^{f(b+3) / h} \ldots$, exactly the pattern claimed.

Corollary 6. $\llbracket n ! \rrbracket \nsupseteq_{p} \llbracket n !-1 \rrbracket$.

Proof. Suppose that $\llbracket n ! \rrbracket \geq_{p} \llbracket n !-1 \rrbracket$. Then by Theorem 5 we obtain

$$
\llbracket n !-1 \rrbracket=u c^{b ! / h} d^{(b+1) ! / h} c^{(b+2) ! / h} d^{(b+3) ! / h} \ldots .
$$

Since in $\llbracket n !-1 \rrbracket$ only groups of 0 s and 1 s occur of increasing size, both $c$ and $d$ either consist only of 0 s or only of 1 s. Since $\llbracket n !-1 \rrbracket$ contains infinitely many 0 s and infinitely many $1 \mathrm{~s}$, either $c$ consists of $0 \mathrm{~s}$ and $d$ consists of $1 \mathrm{~s}$, or the other way around. But then the resulting consecutive groups of 0 s and 1 s have sizes $|c| b ! / h,|d|(b+1) ! / h,|c|(b+2) ! / h),|d|(b+3) ! / h \cdots$, ultimately divisible by any number, which does not hold for the group sizes $n !-1,(n+1) !-1,(n+2) !-1$, $(n+3) !-1 \ldots$ in $\llbracket n !-1 \rrbracket$. This contradiction proves $\llbracket n ! \rrbracket \Varangle_{p} \llbracket n !-1 \rrbracket$.

Corollary 7. $\llbracket n ! \rrbracket \nsupseteq_{p} \llbracket(2 n) ! \rrbracket$.

Proof. As in the previous proof, use the form of the transducts of $\llbracket n$ ! \given by Theorem 5: again $c$ and $d$ both consist of copies of a single symbol, different for the two. But now it will be impossible for such transduct to equal $\llbracket(2 n) ! \rrbracket$ because the growth of the groups in the transduct is like a multiple of $n$ !, which is much slower than that of the groups in $\llbracket(2 n) ! \rrbracket$.

For the same class of functions we now give a characterization for transducts of $\langle f\rangle$ rather than $\llbracket f \rrbracket$. 
Theorem 8. Let $f: \mathbb{N} \rightarrow \mathbb{N}$ be a function for which for every $m>0$ there exists $N \in \mathbb{N}$ such that $f(n) \equiv 0 \bmod m$ for all $n>N$. If $\langle f\rangle \geq_{p} \sigma$ then there exist $k>0, a \geq 0$ and $u, p_{0}, \cdots, p_{k-1}, c_{0}, \cdots, c_{k-1} \in \Sigma^{*}$ such that

$$
\sigma=u \prod_{j=0}^{\infty}\left(\prod_{i=0}^{k-1} p_{i} c_{i}^{f(a+i+j k) / k}\right)=u p_{0} c_{0}^{f(a) / k} p_{1} c_{1}^{f(a+1) / k} \ldots
$$

Proof. Assume that $P(\langle f\rangle)=\sigma$ for a permutation transducer $P=\left(Q, q_{0}, \delta, \lambda\right)$. Choose $k$ by Lemma 2 such that $\delta\left(q, 0^{k}\right)=\delta\left(q, 1^{k}\right)=q$ for all $q \in Q$. By the assumption on $f$ there exists $a$ such that $f(n) \equiv 0 \bmod k$ for all $n \geq a$. Let $v=10^{f(0)} 10^{f(1)} 1 \cdots 10^{f(a-1)}$, which is a prefix of $\langle f\rangle$. Let $u=\lambda\left(q_{0}, v\right)$, and $r_{0}=\delta\left(q_{0}, v\right)$. Define $r_{i}=\delta\left(r_{0}, 1^{i}\right)$ for $i=1,2, \ldots, k$; since $\delta\left(r_{0}, 1^{k}\right)=r_{0}$ we have $r_{k}=r_{0}$. Since $f(a+i) \equiv 0 \bmod k$ we obtain $r_{i+1}=\delta\left(r_{i}, 10^{f(a+i)}\right)$ for $i=0, \ldots, k-1$. Write $p_{i}=\lambda\left(r_{i}, 1\right)$ and $c_{i}=\lambda\left(r_{i+1}, 0^{k}\right)$, then by using $\delta\left(r_{i+1}, 0^{k}\right)=r_{i+1}$ for $i=0, \ldots, k-1$, we obtain the desired result that $\sigma$ equals

$$
u \lambda\left(r_{0}, 10^{f(a)}\right) \lambda\left(r_{1}, 10^{f(a+1)}\right) \lambda\left(r_{2}, 10^{f(a+2)}\right) \cdots=u \prod_{j=0}^{\infty}\left(\prod_{i=0}^{k-1} p_{i} c_{i}^{f(a+i+j k) / k}\right) .
$$

Corollary 9. $\langle n !\rangle \nsupseteq_{p}\langle n !-1\rangle$.

Proof. Suppose that $\langle n !\rangle \geq_{p}\langle n !-1\rangle$. Then by Theorem 8 we obtain

$$
\langle n !-1\rangle=u p_{0} c_{0}^{a ! / k} p_{1} c_{1}^{(a+1) ! / k} p_{2} c_{2}^{(a+2) ! / k} \cdots .
$$

Since in $\langle n !-1\rangle$ only increasing groups of $0 \mathrm{~s}$ occur between consecutive $1 \mathrm{~s}$, every $p_{i}$ contains at most one 1 and every $c_{i}$ only consists of 0 s. By possibly doubling $k$, we may assume that two distinct $p_{i}$ s contain a 1 ; let $p_{g}$ and $p_{h}$ be the first two containing a 1 . For $i=0, \ldots, h-g-1$ define $d_{i}$ by $c_{i}=0^{d_{i}}$. Then for every $j \geq 0$ the string $p_{g} c_{g}^{(a+g+j k) ! / k} p_{g+1} c_{g+1}^{(a+g+1+j k) ! / k} \cdots p_{h}$ is a part of $\langle n !-1\rangle$ containing exactly two $1 \mathrm{~s}$, with exactly $C+\sum_{i=0}^{h-g-1} \frac{(a+g+j k) ! d_{i}}{k}$ separating $0 \mathrm{~s}$, for some constant $C \geq 0$. Choose $N>2 C+2$. Then for $j$ large enough all of these groups of $0 \mathrm{~s}$ have size $C \bmod N$, contradicting the fact that after a finite part $\langle n !-1\rangle$ only contains groups of $0 \mathrm{~s}$ of size $-1 \bmod N$.

Next we switch to functions $f$, like polynomials, for which $n \mapsto(f(n) \bmod m)$ is periodic for every $m>0$. For transducts of $\langle f\rangle$ under permutation transducers the following characterization was given in [2].

Theorem 10. Let $f: \mathbb{N} \rightarrow \mathbb{N}$ be a function for which $n \mapsto(f(n) \bmod m)$ is periodic for every $m>0$. Then $\langle f\rangle \geq_{p} \sigma$ for $\sigma: \mathbb{N} \rightarrow \Sigma$ if and only if there exist $k, h>0$ and $p_{0}, \cdots, p_{k-1}, c_{0}, \cdots, c_{k-1} \in \Sigma^{*}$ such that

$$
\sigma=\prod_{j=0}^{\infty}\left(\prod_{i=0}^{k-1} p_{i} c_{i}^{\lfloor f(i+j k) / h\rfloor}\right)=p_{0} c_{0}^{\lfloor f(0) / h\rfloor} p_{1} c_{1}^{\lfloor f(1) / h\rfloor} \ldots
$$


We give a similar description of transducts of $\llbracket f \rrbracket$.

Theorem 11. Let $f: \mathbb{N} \rightarrow \mathbb{N}$ be a function for which $n \mapsto(f(n) \bmod m)$ is periodic for every $m>0$. If $\llbracket f \rrbracket \geq_{p} \sigma$ for $\sigma: \mathbb{N} \rightarrow \Sigma$ then there exist $k, h>0$ and $p_{0}, \cdots, p_{k-1}, c_{0}, \cdots, c_{k-1} \in \Sigma^{*}$ such that

$$
\sigma=\prod_{j=0}^{\infty}\left(\prod_{i=0}^{k-1} p_{i}\left(c_{i} p_{i}\right)^{\lfloor f(i+j k) / h\rfloor}\right)=p_{0}\left(c_{0} p_{0}\right)^{\lfloor f(0) / h\rfloor} p_{1}\left(c_{1} p_{1}\right)^{\lfloor f(1) / h\rfloor} \cdots,
$$

with the additional constraint that $p_{i}=\epsilon$ if $f(i) \equiv 0 \bmod h$, and $c_{i}$ is non-empty.

The proof follows the same lines as that of Theorem 8, but slightly more technical; for details we refer to the full version [9] of this paper.

\section{Basic Function Operations}

In this section we investigate how $\langle f(n)\rangle$ relates to $\langle f(n+k)\rangle,\langle f(n)+k\rangle,\langle k f(n)\rangle$ and $\langle f(k n)\rangle$, and similarly for $\llbracket \cdot \rrbracket$. For completeness we do not only consider $\sim_{p}, \geq_{p}, \leq_{p}$ based on permutation transducers, but also $\sim, \geq, \leq$ based on ordinary transducers.

Theorem 12. When linear operations on sequences of the form $\langle f\rangle$ or $\llbracket f \rrbracket$ are performed, the general relation between the original sequence and its image by ordinary or permutation is given by an entry in the following two tables:

$\langle f(n)\rangle \leq, \geq, \underline{\leq}_{p}, \geq_{p}\langle f(n)+k\rangle$
$\langle f(n)\rangle \leq, \geq, \underline{\leq}_{p}, ?\langle f(n+k)\rangle$
$\langle f(n)\rangle \leq, \geq, \leq_{p}, \geq_{p}\langle k f(n)\rangle$
$\langle f(n)\rangle \leq, \geq, \underline{\leq}_{p}, \geq_{p}\langle f(k n)\rangle$

$\llbracket f(n) \rrbracket \leq, \geq, \underline{x}_{p}, \geq_{p} \llbracket f(n)+k \rrbracket$
$\llbracket f(n) \rrbracket \leq, \geq, \underline{\leq}_{p}, \geq_{p} \llbracket f(n+k) \rrbracket$
$\llbracket f(n) \rrbracket \leq, \geq, \leq_{p}, \geq_{p} \llbracket k f(n) \rrbracket$
$\llbracket f(n) \rrbracket \leq k, \geq, \underline{\leq}_{p}, \geq_{p} \llbracket f(k n) \rrbracket$

An entry of the form $\sigma \geq \tau$ indicates that for every $f, k$ a transducer exists transforming $\sigma$ to $\tau$, while $\sigma \nsupseteq \tau$ indicates that $f, k$ exist for which such a transducer does not exist, and similar for $\leq, \leq_{p}, \geq_{p}$. The question mark '?' states that this question is open for $\langle f(n)\rangle \geq_{p}\langle f(n+k)\rangle$.

The proofs of all claims can be found in the full version of this paper [9]; for all cases either explicit transducers are given or results from Sect. 3 are applied to show that the transduct does not satisfy the required pattern for a particular function $f$.

\section{Classes of Linear Functions}

For linear polynomial functions $f_{k, l}(n)=k n+l$, the relations between $\left\langle f_{k, l}\right\rangle$ were already dealt with in [2].

Theorem 13. For all $k, l \in \mathbb{N}$ with $k \geq 1:\langle k n+l\rangle \sim_{p}\langle n\rangle$; in other words: all (non-constant) linear functions are equivalent under $\sim_{p}$. 
The situation is markedly different for $\llbracket f_{k, l} \rrbracket$, as we will see in the next theorem. Note that by Proposition 3 and Theorem 4 we already know $\llbracket n \rrbracket<_{p}\langle n\rangle$, so $\langle n\rangle$ is not atomic. We do not yet know whether $\llbracket n \rrbracket$ is atomic or not.

Theorem 14. Under permutation transduction there is an infinite, strictly ascending sequence of equivalence classes containing $\llbracket f \rrbracket$ for linear polynomials $f$, in between $\llbracket n \rrbracket$ and $\langle n\rangle$; in particular

$$
\llbracket n \rrbracket<_{p} \llbracket n+2 \rrbracket<_{p} \llbracket n+4 \rrbracket<_{p} \llbracket n+8 \rrbracket<_{p} \cdots \leq_{p}\langle n\rangle .
$$

Before we give the proof, we state a corollary that settles a question from the previous section.

Corollary 15. No permutation transducer $P$ exists such that $P(\llbracket n+1 \rrbracket)=$ $\llbracket n+2 \rrbracket$.

Proof. This is the content of the first strict inequality in Theorem 14, in combination with $\llbracket n \rrbracket \sim_{p} \llbracket n+1 \rrbracket$. The latter follows from $\overline{\llbracket n+1 \rrbracket}=\llbracket n \rrbracket$, in which $\bar{\sigma}$ denotes the complement of $\sigma$, obtained by permuting the two symbols 0,1 ; it will be clear that $\bar{\sigma}$ can be obtained from $\sigma$ by a one-state permutation transducer.

The proof of Theorem 14 is given in two parts. The first (existence) part is immediate from the following lemma.

Lemma 16. Let $k \geq 0$ be an integer; then $\llbracket n+k \rrbracket \geq_{p} \llbracket n+\left\lfloor\frac{k+1}{2}\right\rfloor \rrbracket$.

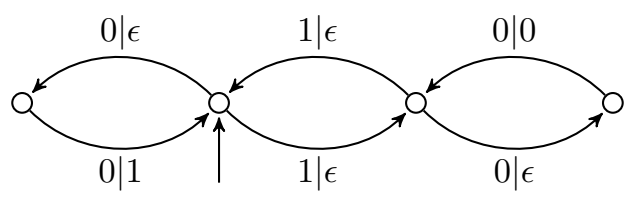

Proof. For $k=0,1$ the statement is trivial; so assume that $k \geq 2$. Consider the four-state permutation transducer given above. For even $k$ it will remove any 1 's from the input sequence $\llbracket n+k \rrbracket$ and alternatingly divide by 2 or divide by 2 and complement, any sequence of 0 's; the result is $\overline{\llbracket n+\frac{k}{2} \rrbracket}$. For odd $k$ it converts $\overline{\llbracket n+k \rrbracket}$ into $\llbracket n+\frac{k+1}{2} \rrbracket$. Taking complements is easily achieved by a permutation transducer, and the permutation property is transitive.

In fact this lemma yields a stronger result than required for Theorem 14, as stated in the following corollary.

Corollary 17. For every $a>0$ we have $\llbracket n+a \rrbracket \geq_{p} \llbracket n \rrbracket$.

Proof. Starting by $\llbracket n+a \rrbracket$ repeat applying Lemma 16 until $\llbracket n+1 \rrbracket$ is obtained. Then the corollary follows from transitivity of $\geq_{p}$ and $\llbracket n+1 \rrbracket \sim_{p} \llbracket n \rrbracket$.

To complete the proof of Theorem 14 we have to prove that for none of the strict steps a backward transduction is possible. This is immediate from the following stronger result. 
Proposition 18. For $a \geq 0, b \geq a+2$ no permutation transducer $P$ exists satisfying $P(\llbracket n+a \rrbracket)=\llbracket n+b \rrbracket$.

Proof. The permutation transducts of $\llbracket n+a \rrbracket$, according to Theorem 11, are of the form

$$
\prod_{j=0}^{\infty}\left(\prod_{i=0}^{k-1} p_{i}\left(c_{i} p_{i}\right)^{\left\lfloor\frac{i+j k+a}{h}\right\rfloor}\right) .
$$

Replace the period $k$ by a multiple (if necessary) in order for $h$ to be a divisor of $k$, and write $m=\frac{k}{h}$ and $a_{i}=\left\lfloor\frac{i+a}{k}\right\rfloor$ for $i=0, \ldots, k-1$. Write $b_{i}=\left\lfloor\frac{i+a}{h}\right\rfloor-$ $a_{i} m$ for $i=0, \ldots, k-1$, note that $0 \leq b_{i}<m$. Then the transduct is of the shape $\prod_{j=0}^{\infty}\left(\prod_{i=0}^{k-1} p_{i}\left(c_{i} p_{i}\right)^{b_{i}+\left(j+a_{i}\right) m}\right)$. Writing $w_{i}=\left(c_{i} p_{i}\right)^{m}$ and replacing $p_{i}$ by $p_{i}\left(c_{i} p_{i}\right)^{b_{i}}$, we conclude that the transduct is of the shape $P(\llbracket n+a \rrbracket)=$ $\prod_{j} \prod_{i=0}^{k-1} p_{i}\left(w_{i}\right)^{j+a_{i}}$.

Now suppose that this image equals $\llbracket n+b \rrbracket$, for some $b \geq a+2$. It is impossible for $w_{i}$ to contain both 0 and 1 , since in that case $\prod_{j} \prod_{i} p_{i} w_{i}^{j+a_{i}}$ contains infinitely many pairs of the same symbol separated by a fixed number of copies of the other symbol, which $\llbracket n+b \rrbracket=0^{b} 1^{b+1} 0^{b+2} 1^{b+3} \ldots$ clearly does not.

Hence each $w_{i}$ consist of copies of a single symbol; if $w_{i+1}$ consists of the same symbol or equals $\epsilon$, we can merge $p_{i} w_{i} p_{i+1} w_{i+1}$ and reduce $k$. Hence without loss of generality we may assume that $w_{i}$ and $w_{i+1}$ consist of different symbols; the same then holds for $p_{i}$ and $p_{i+1}$ (but they could equal $\epsilon$ ). By multiplying the period $k$ we may assume that $k$ is even and $k>b$.

The linear growth of $\llbracket n+b \rrbracket$ implies that each $w_{i}$ will consist of exactly $k$ symbols; since $p_{i} w_{i}$ and $p_{i+1} w_{i+1}$ are consecutive blocks of different symbols, $\# p_{i+1} \bmod k=\left(\# p_{i}+1\right) \bmod k$.

Since $k>b>a$ and $a_{i}=\left\lfloor\frac{i+a}{k}\right\rfloor$ we obtain $a_{i}=0$ for $i<k-a$. So $p_{0} p_{1} \cdots p_{k-a-1}$ is an initial part of $\llbracket n+b \rrbracket$, in which $p_{i}$ alternatingly consist of $0 \mathrm{~s}$ and $1 \mathrm{~s}$. If $p_{0}=\epsilon$ then $p_{1}$ is the first group of $0 \mathrm{~s}$, being $0^{b}$, contradicting $\# p_{i+1} \bmod k=\left(\# p_{i}+1\right) \bmod k$. Hence $p_{0}=0^{b}$, and by $\# p_{i+1} \bmod k=\left(\# p_{i}+\right.$ 1) $\bmod k$ we obtain $\# p_{i}=b+i$ for $i=0,1, \ldots, k-a-1$. But since $\#\left(c_{i} p_{i}\right)=k$ we obtain $\# p_{i} \leq k$. But then we have $b+k-a-1=\# p_{k-a-1} \leq k$, contradicting $b \geq a+2$.

\section{$6 \quad$ Higher Degree Polynomials}

Theorem 19. Let $f, g: \mathbb{N} \rightarrow \mathbb{N}$ be two polynomials of degree $n>1$ with the same leading coefficient such that

- $f-g$ is not constant, and

- $\lim _{x \rightarrow \infty}\left(f(a x)-a^{n} g(x)\right)=\infty$ for every $a>1$.

Then no permutation transducer $P$ exists such that $P(\langle f\rangle)=\langle g\rangle$. 
Proof. Assume that such a $P$ exists. Then according to Theorem 10 there exist $k, h>0$ and $p_{0}, \cdots, p_{k-1}, c_{0}, \cdots, c_{k-1} \in \Sigma^{*}$ such that

$$
\langle g\rangle=\prod_{j=0}^{\infty}\left(\prod_{i=0}^{k-1} p_{i} c_{i}^{\left\lfloor\frac{f(i+j k)}{h}\right\rfloor}\right) .
$$

Since $\lim _{n \rightarrow \infty} g(n)=\infty$, for every $i=0, \ldots, k-1$ no 1 occurs in $c_{i}$, and at most one 1 occurs in $p_{i}$. Since $\langle g\rangle$ contains symbols 1 , at least one of the $p_{i}$ 's contains a symbol 1.

If there is only one such $p_{i}$, by doubling $k$ we make it two.

Let $p_{a}$ and $p_{b}$ be the first two $p_{i}$ 's containing a 1 . Let $q$ be the total number of 1 's in $p_{0}, \ldots, p_{k-1}$, and $c_{i}=0^{a_{i}}$ for $i=0, \ldots, k-1$. Now we count the number of 0 's right after the $q j+1$-th 1 of $\langle f\rangle$ in two ways, and obtain that there is constant $c \geq 0$ (corresponding to the number of 0's occurring in some $p_{i}$ 's) such that

$$
c+\sum_{i=a}^{b-1}\left\lfloor\frac{f(i+j k)}{h}\right\rfloor a_{i}=g(j q)
$$

for all $j \geq 0$.

First we consider the case $k=q$. Then $a=0, b=1$ and we have $c+$ $a_{0}\left\lfloor\frac{f(j k)}{h}\right\rfloor=g(j k)$ for all $j \geq 0$. This is only possible if $f-g$ is constant, which we assumed to be not. In the remaining case we have $0<q<k$.

Write $A=\sum_{i=a}^{b-1} a_{i}$.

Using that $f$ is ascending for sufficiently large arguments, we have $f(j k) \leq$ $f(i+j k) \leq f((j+1) k)$ for $j>C$ for some $C$, and $a \leq i<b$.

Using this and $x-1 \leq\lfloor x\rfloor \leq x$ for all $x$, we obtain

$$
c+A\left(\frac{f(j k)}{h}-1\right) \leq g(j q) \leq c+A \frac{f((j+1) k)}{h}
$$

for all $j \geq C$. Then for $j \rightarrow \infty$ in the above inequalities we obtain $A k^{n}=h q^{n}$. Then the left inequality yields

$$
c-A+\left(\frac{q}{k}\right)^{n} f(j k)=c+A\left(\frac{f(j k)}{h}-1\right) \leq g(j q)
$$

for all $j \geq C$. This contradicts $\lim _{x \rightarrow \infty}\left(f(a x)-a^{n} g(x)\right)=\infty$ for $a=\frac{k}{q}>1$.

Corollary 20. $\left\langle(n+1)^{2}\right\rangle \nsupseteq_{p}\left\langle n^{2}\right\rangle$ and $\left\langle n^{2}\right\rangle \nsupseteq_{p}\left\langle(n-1)^{2}\right\rangle$.

Lemma 21. For $k>0$ there is no permutation transducer $P$ such that $P$ : $\left\langle(n-k)^{2}\right\rangle \mapsto\left\langle(n-2 k)^{2}\right\rangle$.

Proof. Apply Theorem 19 directly to $f=(n-k)^{2}$ and $g=(n-2 k)^{2}$.

Corollary 22. The following provides an infinite ascending chain of quadratic polynomial functions that are non-equivalent under permutation transducers:

$$
\left\langle(n+1)^{2}\right\rangle<_{p}\left\langle n^{2}\right\rangle<_{p}\left\langle(n-1)^{2}\right\rangle<_{p}\left\langle(n-2)^{2}\right\rangle<_{p}\left\langle(n-4)^{2}\right\rangle<_{p}\left\langle(n-8)^{2}\right\rangle<_{p} \cdots .
$$


Proof. Consider the three permutation transducers
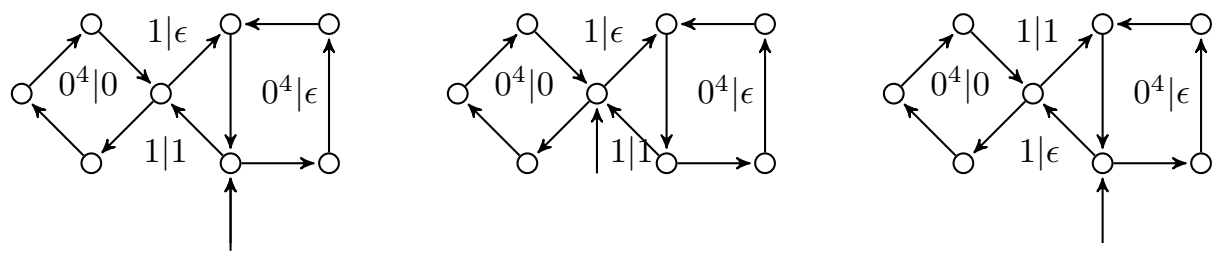

based on the principle that the left cycle reduces $10^{4 m} 1$ to $10^{m}$ and the right cycle $10^{4 m+1} 1$ to 1 . Here $0^{4} \mid u$ means that all four arrows consume 0 , while only one has output $u$, the others have empty output. It is not hard to see that the first transduces $\left\langle(n-2 k)^{2}\right\rangle$ to $\left\langle(n-k)^{2}\right\rangle$ for every $k>0$, the second transduces $\left\langle(n-1)^{2}\right\rangle \mapsto\left\langle n^{2}\right\rangle$ and the third transduces $\left\langle n^{2}\right\rangle$ to $\left\langle(n+1)^{2}\right\rangle$. None of the arrows is reversible by Corollary 20 and Lemma 21.

Remark 23. Now consider the permutation transducers:
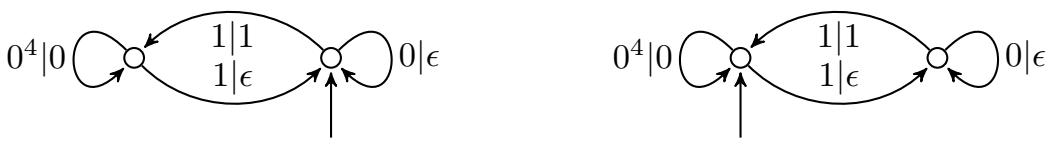

The one on the left (or the first of the three transducers in the previous picture) is easily seen to provide transition from $\left\langle(n+2 k)^{2}\right\rangle$ to $\left\langle(n+k)^{2}\right\rangle$, for $k>0$, so

$$
\left\langle(n+1)^{2}\right\rangle \leq_{p}\left\langle(n+2)^{2}\right\rangle \leq_{p}\left\langle(n+4)^{2}\right\rangle \leq_{p}\left\langle(n+8)^{2}\right\rangle \leq_{p} \cdots ;
$$

but here transductions in the opposite direction are not ruled out by Theorem 19. The other transducer shows that $\left\langle(n+2 k-1)^{2}\right\rangle \geq p\left\langle(n+k)^{2}\right\rangle$ for $k>0$, and puts $\left\langle(n+2 k-1)^{2}\right\rangle$ in some infinite non-descending sequence. For example:

$$
\left\langle(n+1)^{2}\right\rangle \leq_{p}\left\langle(n+2)^{2}\right\rangle \leq_{p}\left\langle(n+3)^{2}\right\rangle \leq_{p}\left\langle(n+5)^{2}\right\rangle \leq_{p}\left\langle(n+9)^{2}\right\rangle \cdots .
$$

\section{References}

1. Allouche, J.P., Shallit, J.: Automatic Sequences: Theory, Applications, Generalizations. Cambridge University Press, Cambridge (2003)

2. Bosma, W., Zantema, H.: Ordering sequences by permutation transducers. Indagationes Math. 38, 38-54 (2017)

3. Endrullis, J., Grabmayer, C., Hendriks, D., Zantema, H.: The degree of squares is an atom. In: Manea, F., Nowotka, D. (eds.) WORDS 2015. LNCS, vol. 9304, pp. 109-121. Springer, Cham (2015). doi:10.1007/978-3-319-23660-5_10

4. Endrullis, J., Hendriks, D., Klop, J.W.: Degrees of streams. Integers Electron. J. Comb. Number Theor. 11B(A6), 1-40 (2011). Proceedings of Leiden Numeration Conference (2010)

5. Endrullis, J., Karhumäki, J., Klop, J.W., Saarela, A.: Degrees of infinite words, polynomials and atoms. In: Brlek, S., Reutenauer, C. (eds.) DLT 2016. LNCS, vol. 9840, pp. 164-176. Springer, Heidelberg (2016). doi:10.1007/978-3-662-53132-7_14 
6. Endrullis, J., Klop, J.W., Saarela, A., Whiteland, M.: Degrees of transducibility. In: Manea, F., Nowotka, D. (eds.) WORDS 2015. LNCS, vol. 9304, pp. 1-13. Springer, Cham (2015). doi:10.1007/978-3-319-23660-5_1

7. Pin, J.-E.: On reversible automata. In: Simon, I. (ed.) LATIN 1992. LNCS, vol. 583, pp. 401-416. Springer, Heidelberg (1992). doi:10.1007/BFb0023844

8. Sakarovitch, J.: Elements of automata theory. Cambridge University Press, Cambridge (2009)

9. Zantema, H., Bosma, W.: Extended version of this paper (2017). http://www.win. tue.nl/ hzantema/permtr.pdf 